\title{
Automatic Extraction of Requirements from State-based Hardware Designs for Runtime Verification
}

\author{
Minjun Seo and Roman Lysecky \\ Department of Electrical and Computer Engineering, University of Arizona, Tucson, AZ, USA \\ mjseo@email.arizona.edu, rlysecky@ece.arizona.edu
}

\begin{abstract}
Runtime monitoring and verification enables a system to monitor itself and ensure system requirements are met even in the presence of dynamic environments. For hardware, state-based models are widely used, but verifying the correctness between the state-based model and hardware implementation is timeconsuming and difficult. This paper presents a novel method for extracting hardware verification requirements from state-based hardware models to construct a hierarchical runtime monitoring graph that can be efficiently used at runtime to verify correctness.
\end{abstract}

\section{CCS CONCEPTS}

- Hardware Model checking • Computing methodologies Model verification and validation

\section{KEYWORDS}

Runtime verification, formal requirements models, periodic statement machines, hardware-based observations.

\section{ACM Reference format:}

Minjun Seo and Roman Lysecky. 2019. Automatic Extraction of Requirements from State-based Hardware Designs for Runtime Verification. In GLSVLSI '19: 2019 Great Lakes Symposium on VLSI, May 9-11, 2019, Washington, D.C., USA. ACM, New York, NY. 4 pages. DOI: https://doi.org/10.1145/3299874.3318021

\section{INTRODUCTION}

Defining and verifying system requirements is a challenging task in embedded systems. Test-based verification and formal verification comprise a considerable effort that accounts for $75 \%$ of the total system design effort [4]. However, despite existing efforts, complete system verification at design time is considered to be almost impossible due to system complexity, dynamic execution behaviors, and unpredictable system inputs.

Runtime monitoring and verification enables a system to monitor its own execution to support rapid in-situ failure detection and enables tracing a failure's root cause, especially root causes that arise due to unknown environmental, physical, and operating conditions. However, cost, size, area, and energy constraints often limit which monitoring and verification

This research was partially supported by the National Science Foundation under Grant CNS-1563652.

Permission to make digital or hard copies of all or part of this work for personal or classroom use is granted without fee provided that copies are not made or distributed for profit or commercial advantage and that copies bear this notice and the full citation on the first page. Copyrights for components of this work owned by others than ACM must be honored. Abstracting with credit is permitted. To copy otherwise, or republish, to post on servers or to redistribute to lists, requires prior specific permission and/or a fee. Request permissions from Permissions@acm.org.

GLSVLSI '19, May 9-11, 2019, Tysons Corner, V A, USA

(C) 2019 Association for Computing Machinery.

ACM ISBN 978-1-4503-6252-8/19/05 ..\$15.00

https://doi.org/10.1145/3299874.3318021 approaches can be employed. A key concern is the need to ensure the monitoring method does not change the observable system state and to avoid any timing changes. Such intrusive interactions could lead to synchronization and scheduling problems that then lead to catastrophic system failures. Thus, runtime monitoring and verification methods should be non-intrusive, especially for safety-critical and life-critical embedded systems.

Embedded systems typically require tight interactions and interfacing between software and hardware components. Many embedded systems are implemented as systems-on-a-chip (SOC) that have a large number of hardware components. Verifying embedded systems at runtime not only includes verifying software execution, but also verifying the correct execution of hardware components, and interactions thereof. Thus, runtime monitoring and verification methods must also be able to monitor and verify the execution of hardware components.

In this paper, we present an extension of our runtime software requirements monitoring framework [10] to incorporate nonintrusive in-situ requirements monitoring for state-based hardware designs (NIRM-H) in embedded systems. We present a methodology for extracting hardware verification requirements from state-based hardware models to construct a hierarchical runtime monitoring graph (HRMG). To support automated synthesis of requirements for state-based hardware description, a formal model that extends the concept of periodic state machines with explicitly defined data transfer/synchronization is employed. The synthesized HRMG is a compact model of the system requirements that can be monitored at runtime using on-chip hardware, which can efficiently monitor the system execution with no performance overhead.

\section{RELATED WORK}

Runtime monitoring for hardware can be utilized to dynamically verify that behaviors of a system's hardware components adhere to the required system properties. However, most monitoring methods for hardware are not specifically targeted at system-level in-situ and runtime verification, but rather target the monitoring of specific operations or target verification/testing before system deployment. Trace-based methods for runtime monitoring (e.g., ChipScope [2], MED [6]) use large volumes of real-time trace data for analysis, which allow only a very simple level of verification, such as specifying a trigger with a simple condition. Event-based methods (e.g., ARBD [8], BusMOP [7], Heffernan et al. [5]) use hardware to detect events. Thus, event-based monitoring requires analyzing less data and results in simpler implementations that enable on-chip verification. Formal models, such as timed automata (TA) [1] and linear temporal logic (LTL) [9], are useful 


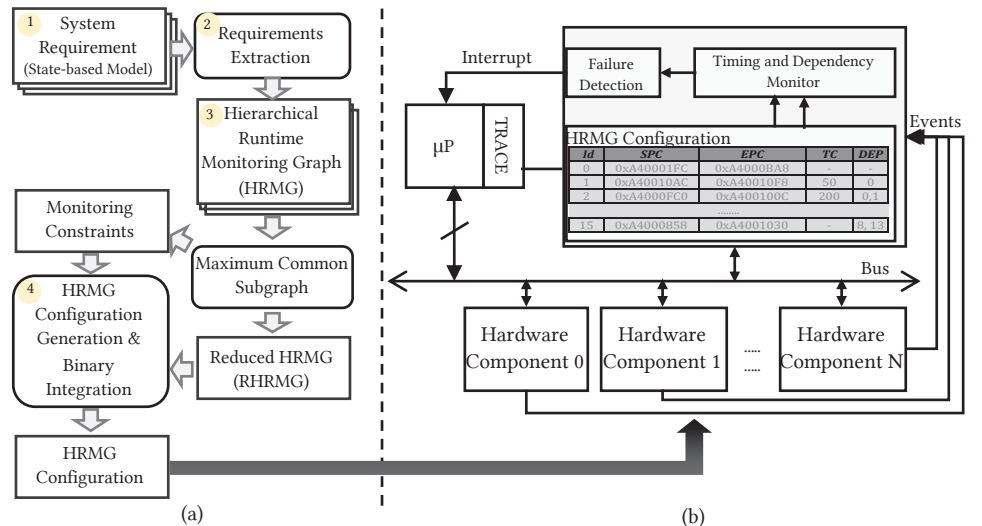

Figure 1: Non-Intrusive In-situ Requirements Monitoring methodology.

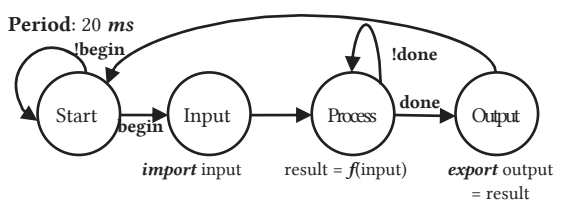

Figure 2: Example periodic state machine.

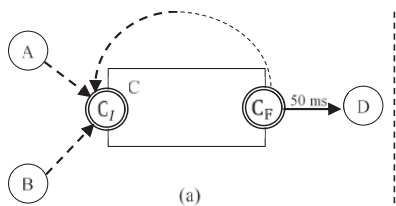

Figure 3: a) Top-level HRMG including hierarchical event $C$, and $b$ ) internal events and dependencies for hierarchical event $C$. for verification, but these approaches require that system designers specify requirements in a separate model that is conceptually disjoint from the hardware design itself.

\section{NIRM METHODOLOGY}

Figure 1 (a) presents an overview of the non-intrusive requirements monitoring (NIRM) methodology consisting of: 1) initial hardware development with state-based models, 2) automated synthesis of requirements from the state-based model, 3) construction of a hierarchical runtime monitoring graph (HRMG) for each state machine, integration and simplification of the HRMGs using maximum common subgraph algorithm, and 4) generation of the configuration that will be programmed within the NIRM hardware. Figure 1 (b) presents an overview of the integration of the in-situ, non-intrusive monitoring hardware that verifies requirements at runtime within the deployed system.

\subsection{Periodic State Machine (PSMs)}

For verifying state-based hardware components, we assume a formal definition of the hardware model exists, or the hardware description itself enables the automatic extraction to create the hardware model. In our approach, we integrate concepts of periodic finite state machines with explicit definitions of data communication and synchronization between state machines in hardware and between hardware and software components.

Definition 1. A periodic state machine (PSM) is a tuple $\mathrm{G}=$ $<P, S, S_{0}, \delta, I M, E X>$ where:

- $\mathrm{P}$ is the period at which the state machine executes. The period $\mathrm{P}$ is defined as an explicit time unit, e.g. $200 \mathrm{~ns}$

- $\mathrm{S}$ is a finite and non-empty set of vertices, where each vertex represents a state. A state is fired at multiple of $\mathrm{P}$ time units.

- $\mathrm{S}_{0}$ is an initial state, an element of $\mathrm{S}$

- $\delta$ is the state-transition function: $\delta: S \times \Sigma \rightarrow S$ where $\Sigma$ is a transition condition.

- IM is a set of shared variables from other PSMS that are imported into this PSM

- EX is a set of shared variables that are exported from this PSM that can be imported to other PSMs

Definition 2. A state $S$ in a PSM $G$ is a tuple $S=\left\langle V_{i}, A, V e\right\rangle$ where:

- $\mathrm{V}_{\mathrm{i}}$ is a shared variable in a state, $\mathrm{V}_{\mathrm{i}} \in \mathrm{I}$

- $V_{e}$ is a shared variable in a state, $V_{e} \in E$

- A is a set of actions (statements) executed in state $S$

If a state needs to access data exports from another PSM, the
PSM model requires the explicit access of that data using an import statement. Similarly, if the PSM needs to output data to be communicated to another PSM, an explicit export is required.

Figure 2 shows a graphical representation of a periodic state machine (PSM) that has a $20 \mathrm{~ms}$ period, 4 states, 3 statements, 1 import variable, and 1 export variable.

\subsection{Runtime Monitoring Model}

We define a hierarchical runtime monitoring graph (HRMG) that is constructed from the state-based PSM models, which captures all requirements that can be monitored. The HRMG defines the set of events that is observable at runtime, the execution orders of those events, and timing constraints between events. All communication (i.e., import and export) in the state-based models are considered system events.

Definition 3. An HRMG is a tuple $\mathrm{G}=\left\langle V_{G}, E_{G}\right\rangle$ where:

- $\mathrm{V}_{\mathrm{G}}$ is a finite set of vertices, where each vertex represents a system event.

- $\mathrm{E}_{\mathrm{G}}$ is a set of edges representing dependencies between vertices. Each edge $E \in E_{G}$, defined as $E=V_{G} \rightarrow V_{G}{ }^{\prime}$, represents a dependency between two events $V_{G}$ and $V_{G}{ }^{\prime} . V_{G}$ is the source event, and $\mathrm{V}_{\mathrm{G}}{ }^{\prime}$ is the sink event.

- If a deterministic time constraint exists between $\mathrm{V}_{\mathrm{G}}$ and $\mathrm{V}_{\mathrm{G}}{ }^{\prime}$, an edge $\mathrm{E}$ may specify a worst-case execution time (WCET) constraint, defined as $V_{G} \stackrel{\text { time }}{\longrightarrow} V_{G}{ }^{\prime}$. Note that the WCET can be obtained by period or probabilistic analysis.

- An edge has a type $\in$ \{strong, weak\} that specifies if the dependency is mandatory or optional. strong (solid arrow) represents a mandatory dependency. weak (dashed arrow) represents an optional dependency.

- A hierarchical event $\mathrm{H} \in \mathrm{V}_{\mathrm{G}}$ is defined by a tuple $\mathrm{H}=$ $\left\langle V_{H}, E_{H}, H_{I}, H_{F}\right\rangle . H_{I} \in V_{H}$ is the initial vertex for $H$. External edges to $\mathrm{H}$ connect only to $\mathrm{H}_{\mathrm{I}}$. $\mathrm{H}_{\mathrm{F}} \in \mathrm{V}_{\mathrm{H}}$ is the final vertex for $\mathrm{H}$. External edges from $\mathrm{H}$ only originate from $\mathrm{H}_{\mathrm{F}}$.

- For a hierarchical event $\mathrm{H}$, all incoming edges to $\mathrm{H}_{\mathrm{I}}$ must have the same edge type (i.e., incoming edges must all be strong or weak dependencies).

Figure 3 presents an illustrative example of the HRMG model. Figure 3 (a) shows the top-level view of a graph consisting of 5 events, one of which is a hierarchical event, 3 weak dependencies, and 1 strong dependency. The hierarchical event $\mathrm{C}$ graphically depicts the initial $\left(\mathrm{C}_{\mathrm{I}}\right)$ and final $\left(\mathrm{C}_{\mathrm{F}}\right)$ events. $\mathrm{C}_{\mathrm{I}}$ has three incoming edges from $\mathrm{A}, \mathrm{B}$, and $\mathrm{C}_{\mathrm{F}}$, all of which represent weak dependencies. Figure 3 (b) shows the events and dependencies within hierarchical event $\mathrm{C}$, which consists of a total of 5 events, including the initial $\left(\mathrm{C}_{\mathrm{I}}\right)$ and final $\left(\mathrm{C}_{\mathrm{F}}\right)$ events. The edge $\mathrm{CF} \stackrel{50 \mathrm{~ms}}{\longrightarrow} \mathrm{D}$ 
indicates that event $\mathrm{D}$ should occur no more than $50 \mathrm{~ms}$ after $\mathrm{C}_{\mathrm{F}}$.

\subsection{Model Transform (PSM to HRMG)}

The PSM represents the state-based hardware model, whereas the HRMG defines the requirements to be monitored at runtime. To create the HRMG, a PSMs-to-HRMG algorithm is utilized to extract requirements from each PSM, which are then combined into a single HRMG for the system-level hardware verification.

To extract the essential data communication, the algorithm first determines the subsets of import states and export states. For each pair of import states and export states, the algorithm analyzes the execution paths between the import-to-export and export-to-export state to determine both execution sequence dependencies and timing constraints. The algorithm traverses the PSM to count the number of intermediate states between the import and export state. If a finite number of states are encountered, a time constraint can be defined by the length of state sequence and PSM period. If the intermediate states have a cyclic path, the timing constraints will be infinite $(\infty)$. The algorithm constructs the corresponding events, and timing constraint if non-infinite, in the HRMG. When constructing the dependencies between events the HRMG, if the in-degree of any node is greater than 1, it needs to change all incoming edges to weak dependency. Finally, the algorithm uses a maximum common subgraph (MCS) algorithm [3] to identify common operation sequences in the RMGs to create hierarchal events. The MCS algorithm finds the largest completely connected subgraph starting with an event and ending with an event, creating a hierarchical event for the subgraph [10]. The MCS is iteratively applied to until no new hierarchical events can be found. When converting subgraphs to hierarchical events, edges to the hierarchical events are converted to weak dependencies as multiple executions may now occur before the hierarchical event, but not all events must occur.

\section{NIRM HARDWARE}

We developed a Xilinx Artix-7 FPGA (XC7A200T)-based prototype of a typical embedded system architecture incorporating the NIRM-H implementation. The NIRM hardware supports monitoring hardware components and verifying the requirements defined within an HRMG.

Multiple events from multiple hardware components must be observed at each hardware component, but those observed events are analyzed at the single NIRM component. As such, an event trace framework is used to efficiently communicate the observed events from each hardware component to the NIRM core. To support this, events detected at each hardware core are encoded using a 5-bit ComponentId, 5-bit EventId, and 32-bit Timestamp. A NIRM Core Wrapper is integrated with each hardware component and uses an internal timer to determine the instance at which a local hardware event is observed. The NIRM Core Wrapper then transmits the timestamp of the local timer with the event's EventId and the component's ComponentId. A NIRM-H Controller receives events from all NIRM Core Wrappers and implements a priority queue that forwards the incoming event with the smallest timestamp to the NIRM hardware each clock cycle. This ensures the NIRM component
Table 1: Area, power consumption, and percentage overhead for NIRM-H compared to a base system.

\begin{tabular}{c|c|cc}
\hline \multirow{2}{*}{$\begin{array}{c}\text { Monitored } \\
\text { Components }\end{array}$} & \multirow{2}{*}{$\begin{array}{c}\text { Power } \\
\text { (\% overhead) }\end{array}$} & $\begin{array}{c}\text { LUTs } \\
\text { (\% overhead) }\end{array}$ & $\begin{array}{c}\text { FF } \\
\text { (\% overhead) }\end{array}$ \\
\hline \hline 4 & $0.145 \mathrm{~W}(4.04 \%)$ & $168(1.5 \%)$ & $48(0.5 \%)$ \\
\hline 8 & $0.146 \mathrm{~W}(4.07 \%)$ & $410(3.5 \%)$ & $136(1.3 \%)$ \\
\hline 16 & $0.148 \mathrm{~W}(4.12 \%)$ & $778(6.4 \%)$ & $312(2.9 \%)$ \\
\hline 32 & $0.150 \mathrm{~W}(4.18 \%)$ & $1640(12.6 \%)$ & $692(6.2 \%)$ \\
\hline 64 & $0.157 \mathrm{~W}(4.36 \%)$ & $3385(22.9 \%)$ & $1486(12.4 \%)$ \\
\hline
\end{tabular}

processes observed events in the correct execution order, even though the events will arrive at different times.

Our methodology uses a hardware-based, on-chip monitor to ensure the system will not suffer any performance degradation. System designers must determine the maximum number of events to monitor when integrating the NIRM hardware. Table 1 presents the hardware area (LUTs and FFs), power consumption, and percentage area and power overhead (compared to a base system) for increasing number of monitored hardware components. Table 1 also shows the area and the power consumption scales linearly.

\section{EXPERIMENTAL RESULTS}

\subsection{Representative PSM Models}

To evaluate the PSM to HRMG synthesis, we developed 7 representative PSM models with increasing complexity. Table 2 summarizes the number of imports, exports, and states for each of PSMs, reporting the number of events, the number of strong and weak dependencies, and the number of timing requirements extracted. While the full specification of the representative PSMs is not shown for brevity, the following notes key features of each and their effect on the HRMG synthesis.

In PSM1, an export-to-export path exists. But due to 2 cyclic paths, the PSM to HRMG synthesis cannot determine a precise time constraint for the export-to-export path, so only a sequence dependency is generated. In PSM2, three import-to-export pairs and one export-to-export pair exists. The PSM to HRMG synthesis is able to determine time constraints for those paths, which are integrated into the resulting HRMG. PSM3 is similar to PSM2 but contains a cyclic path between states, so a precise timing constraint cannot be determined because of the cyclic path. PSM 4 - PSM 7 include multiple PSMs that communicate with each other. In PSM4, a state exports a shared variable that is imported by the other PSM in another state. This implies that the latter state should execute after the former state, the requirement of which is synthesized in the HRMG. In the resulting HRMG, an event has two weak dependencies to itself and to the event related to import according to the principle of handling OR relationships in HRMG. PSM5 contains two independent PSMs. When

Table 2: HRMG requirements synthesis results.

\begin{tabular}{|c|c|c|c|c|c|c|c|}
\hline \multirow{2}{*}{ ID } & \multirow{2}{*}{ Import } & \multirow{2}{*}{ Export } & \multirow{2}{*}{ State } & \multirow{2}{*}{ \# Events } & \multicolumn{2}{|c|}{ Dependencies } & \multirow{2}{*}{$\begin{array}{c}\text { Timing } \\
\text { Constraints }\end{array}$} \\
\hline & & & & & Strong & Weak & \\
\hline PSM1 & 0 & 1 & 3 & 1 & 1 & - & - \\
\hline PSM2 & 3 & 1 & 5 & 4 & - & 3 & 2 \\
\hline PSM3 & 3 & 1 & 6 & 3 & - & 2 & - \\
\hline PSM4 & 3 & 2 & 9 & 4 & 2 & 2 & 1 \\
\hline PSM5 & 4 & 2 & 9 & 8 & 4 & 7 & 2 \\
\hline PSḾ & 2 & 3 & 10 & 7 & 1 & 8 & 1 \\
\hline PSM & 4 & 4 & 17 & 10 & 4 & 14 & 1 \\
\hline $\mathrm{CA}$ & 9 & 8 & 20 & 13 & 1 & 19 & 11 \\
\hline
\end{tabular}




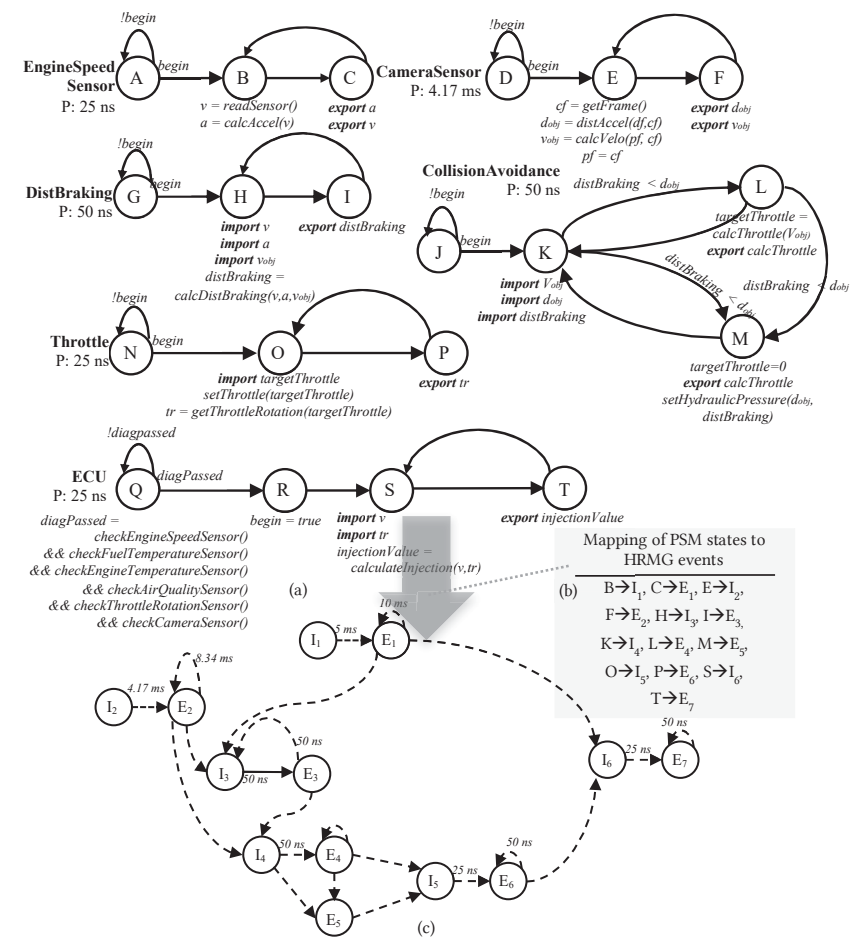

Figure 4: (a) PSM model for collision avoidance system, (b) mapping information, and (c) corresponding HRMG. Note: These diagrams are intended to highlight the characteristic and complexity of the system, so details may not be visible.

independent HRMG are combined, a source and sink node are added to merge the HRMG into a single HRMG, but no other dependencies exist. In PSM6, two of the PSMs have a shared variable, and the third PSM is independent of the other two. PSM7 has two PSMs, in which the second PSM has an import-to-export pair, and the time between the two is deterministic. Thus, a timing constraint is constructed for the event in the HRMG.

\subsection{Case Study: Collision Avoidance}

We further evaluate a case study of a safety-critical embedded system. Figure 4 (a) presents the PSMs for a video-based collision avoidance (CA) system. This implementation uses a single frontmounted camera to detect the location, distance, and speed of cars traveling in the same lane as the vehicle. The CA system calculates the minimum safe following distance and applies the brakes (and reduces throttle) automatically if the distance is less than the minimum safe distance.

The CA system consists of six PSMs. The EngineSpeedSensor PSM has a 25 ns period, which is the half the period at which the vehicle speed sensor is sampled. The EngineSpeedSensor PSM determines the velocity, $\boldsymbol{v}$, computes the vehicles acceleration, $\boldsymbol{a}$, and exports both to other PSMs. The CameraSensor PSM records video at 120 frames per second, which results in a period of 4.17 ms, and calculates the values $\boldsymbol{d}_{\boldsymbol{o b j}}$ and $\boldsymbol{v}_{\boldsymbol{o b j}}$, which are the distance to and speed of the vehicle in front of the current vehicle, respectively, using the current $(\boldsymbol{c} f)$ and previous frames $(p f)$, which are exported in the next state, F. The DistBraking PSM determines the safe braking distance. In state $\mathrm{H}$, the PSM imports the current vehicle speed $(\boldsymbol{v})$, current vehicle acceleration $(\boldsymbol{a})$, and speed of the vehicle in front $\left(v_{o b j}\right)$ and calculates the minimum safe traveling distance using the calcDistBraking function. The resulting distance, distBraking, is exported in state $\mathrm{I}$. The CollisionAvoidance PSM determines the throttle or braking required to maintain the minimum safe distance based on the distance to the vehicle in front $\left(V_{o b j}\right)$, speed of the vehicle $\left(d_{o b j}\right)$, and safe braking distance (distBraking). The Throttle PSM adjusts the actual throttle based on the current throttle position, throttleRotation, and the imported target throttle, targetThrottle. Lastly, the ECU is an integrated controller operating at $40 \mathrm{MHz}$ that imports velocity and throttle positions to control engine operations. Note the ECU model has been simplified for demonstration.

Figure 4 (c) presents the resulting HRMG for the CA system and (b) lists the mapping between PSM states and HRMG events for the case study, the resulting HRMG consists of 13 events, 1 strong dependency, 19 weak dependencies, and 11 timing constraints. In addition, the case study shows a tendency to have more weak dependencies in the result than strong dependencies, which implies that the relationship between the component PSMs is tightly coupled.

\section{CONCLUSIONS}

We presented a non-intrusive in-situ requirements monitoring methodology for hardware designs for automatically extracting requirements and constructing HRMG from state-based hardware designs. Using both a set of representative state models of increasing complexity and a case study of a video-based collision avoidance system, we demonstrated the automated PSM to HRMG requirements synthesis process. Using efficient on-chip hardware, the NIRM approach can then monitor the execution of numerous interacting hardware components at runtime to ensure system requirements are not violated, and automatically reporting any such violations.

\section{REFERENCES}

[1] Alur, R. 1999. Timed Automata. Computer Aided Verification. Springer, Berlin, Heidelberg. 8-22.

[2] ChipScope Pro Software and Cores: 2012. https://www.xilinx.com/support/documentation/sw_manuals/xilinx14_7/c hipscope_pro_sw_cores_ug029.pdf.

[3] Durand, P.J., Pasari, R., Baker, J.W. and Tsai, C. 1999. An efficient algorithm for similarity analysis of molecules. Internet fournal of Chemistry. 2, 17 (1999), $1-16$.

[4] Hailpern, B. and Santhanam, P. 2002. Software debugging, testing, and verification. IBM Systems fournal. 41, 1 (2002), 4-12.

[5] Heffernan, D. and Macnamee, C. 2016. Runtime observation of functional safety properties in an automotive control network. Journal of Systems Architecture. 68, (2016), 38-50. DOI:https://doi.org/10.1016/j.sysarc.2016.05.001.

[6] Leatherman, R. and Stollon, N. 2005. An embedding debugging architecture for SOCs. IEEE Potentials. 24, 1 (Feb. 2005), 12-16. DOI:https://doi.org/10.1109/MP.2005.1405795.

[7] Pellizzoni, R., Meredith, P., Caccamo, M. and Roşu, G. 2008. Hardware runtime monitoring for dependable COTS-based real-time embedded systems. Proceedings - Real-Time Systems Symposium. (2008), 481-491. DOI:https://doi.org/10.1109/RTSS.2008.43.

[8] Peterson, K. and Savaria, Y. Assertion-based on-line verification and debug environment for complex hardware systems. 2004 IEEE International Symposium on Circuits and Systems (IEEE Cat. No.04CH37512) II-685-8.

[9] Pnueli, A. 1977. The temporal logic of programs. 18th Annual Symposium on Foundations of Computer Science (sfcs 1977) (Sep. 1977), 46-57.

[10] Seo, M. and Lysecky, R. 2017. Hierarchical non-intrusive in-situ requirements monitoring for embedded systems. (2017), 259-276. 